\title{
Ablation Monitoring with Elastography: 2D In-vivo and 3D Ex-vivo Studies
}

\author{
Hassan Rivaz ${ }^{\star}$ Ioana Fleming, Lia Assumpcao, Gabor Fichtinger, \\ Ulrike Hamper, Michael Choti, Gregory Hager, and Emad Boctor
}

ERC for Computer Integrated Surgery, Johns Hopkins University. Dept. of Surgical Oncology, Johns Hopkins Medicine. School of Computing, Queens University $\{$ rivaz,inicola1\}@jhu.edu, eboctor $1 @ j h m i . e d u$

\begin{abstract}
The clinical feasibility of $2 \mathrm{D}$ elastography methods is hindered by the requirement that the operator avoid out-of-plane motion of the ultrasound image during palpation, and also by the lack of volumetric elastography measurements. In this paper, we develop and evaluate a 3D elastography method operating on volumetric data acquired from a 3D probe. Our method is based on minimizing a cost function using dynamic programming (DP). The cost function incorporates similarity of echo amplitudes and displacement continuity. We present, to the best of our knowledge, the first in-vivo patient studies of monitoring liver ablation with freehand DP elastography. The thermal lesion was not discernable in the B-mode image but it was clearly visible in the strain image as well as in validation CT. We also present 3D strain images from thermal lesions in ex-vivo ablation. Good agreement was observed between strain images, CT and gross pathology.
\end{abstract}

\section{Introduction}

Hepatocellular carcinoma (HCC) is one of the most common tumors, causing 662,000 deaths worldwide annually. Minimally invasive RF ablation [1] has gained much interest recently since only $10 \%$ to $20 \%$ of patients with HCC are amenable to traditional therapy of surgical resection of the tumor. In RF ablation, an electrode is placed into the tumor to cauterize it [1]. Monitoring the ablation process in order to document adequacy of margins during treatment is a significant importance. Ultrasonography is the most common modality for both target imaging and for ablation monitoring. However, ultrasonographic appearance of ablated tumors only reveals hyperechoic areas due to microbubbles and outgasing and cannot adequately visualize the margin of tissue coagulation.

Accordingly, ultrasound elastography (Ophir et al, 1991) has emerged as a useful augmentation to conventional ultrasound imaging. Elastography has been used for monitoring RF ablation 2, 3] by observing that ablated region is harder than surrounding tissue. In the most common variation of elastography, ultrasound images are captured while the tissue is being compressed, and images

\footnotetext{
* Supported by the Link Foundation Fellowship.
} 
are processed to provide a grid of local displacement measurements. These displacement fields are then used to determine the elastic properties of the tissue at each grid location. The grid of calculated elastic properties can be displayed as an image.

Elastography is computationally expensive, making it challenging to display strain images in real-time. Real-time feedback, however, is required for image guided ablation operations. Another aspect is that signal decorrelation between the pre- and post-compression images induces significant noise in the obtained displacement map and is one of the major limiting factors in elastography [4. Methods based on cross-correlation and phase zero estimation are currently the most popular real-time elastography techniques which provide fast and accurate motion tracking. In RF ablation, however, high decorrelation between pre- and post-compression images results in high noise in the strain images obtained using cross-correlation [3]. Phase zero estimation methods require an estimate of the center frequency of the ultrasound RF signal, which varies with depth due to frequency-dependent attenuation in tissue [5]. This variation can be significant in RF ablation, leading to poor displacement estimation [5].

We have recently developed a real-time 2D elastography method based on dynamic programming (DP) [6]. The method is more robust to signal decorrelation than standard cross-correlation methods and is therefore a good candidate for ablation monitoring where being real-time and robustness to noise are critically important. Here we report, to the best of our knowledge, the first in-vivo patient results on monitoring RF ablation with 2D DP elastography and corroborate the results with CT scans. As initial clinical studies revealed limitations of $2 \mathrm{D}$ elastography in monitoring the thermal ablation, we were compelled to progress toward 3D. We think the readership will find it informative to see how our concept and methodology evolved. We extend our DP method to operate on $3 \mathrm{D}$ volumes. The benefits of $3 \mathrm{D}$ strain imaging of RF ablation are two-fold: 1) 3D imaging eliminates the need to image the same plane while palpating the tissue, which can be very difficult in the presence of breathing and cardiac motion, and 2) 3D imaging allows more precise monitoring of temperature deposition which exhibits variations in $3 \mathrm{D}$, particularly in the presence of blood vessels which act as heat sinks. Previous work has generated 3D elastography by moving a conventional 2D probe out-of-plane using mechanical guidance [78] or freehand [9]. In recent work by Treece et al. 10 and Fisher et al. 11] a 3D probe is used to acquire $3 \mathrm{D}$ elastogrophy, using phase zero and cross-correlation based motion tracking methods respectively. Here, we use 3D probe to acquire 3D data and introduce a 3D DP motion tracking algorithm. We show that 3D elastography can be successfully used to monitor ablation in $3 \mathrm{D}$.

\section{3D Displacement Estimation Using DP}

Compared to other optimization techniques, DP is an efficient non-iterative method of global optimization [1213. We have recently developed a real-time 
2D elastography method using DP [6]. In DP elastography, a cost function which incorporates similarity of echo amplitudes and displacement continuity is minimized. Since data alone can be insufficient to solve ambiguities of motion tracking due to signal decorrelation, the physical priors of tissue motion continuity increases the robustness of the technique 6]. We have showed that DP generates high quality strain images of freehand palpation elastography with up to $10 \%$ compression, indicating that the method is more robust to signal decorrelation (caused by scatterer motion in high axial compression and non-axial motions of the probe) in comparison to the standard correlation techniques. The method operates in less than $1 \mathrm{sec}$ and is thus also suitable for real time elastography.

Here, we extend this method to operate on $3 \mathrm{D}$ volumes. Devising a DP algorithm for optimization involves:

1. Breaking the total optimization cost into a sum of individual costs, such that each cost corresponds to a discrete decision. The decisions should follow each other sequentially and the cost corresponding to each decision should only depend on the previous and not the future decisions (causality).

2. Determining what decisions are possible at each stage.

3. Writing a recursion on the optimal cost from the first stage to the final stage.

Let $g_{j}^{k}(i)$ be the intensity of the $i^{t h}$ sample (axial direction), $j^{\text {th }}$ A-line (lateral direction) and $k^{t h}$ frame (out-of-plane direction) of the pre-compression ultrasound volume. Let $g_{j+d_{l}}^{k+d_{e}{ }^{\prime}}\left(i+d_{a}\right)$ correspond to the post-compression volume where $d_{a}, d_{l}$ and $d_{e}$ represents axial, lateral and elevational displacements respectively, and the size of the volume be $m \times n \times p$. The difference between the two signals, $\Delta$, can be quantified using sum of absolute differences (SAD), which is computationally inexpensive to compute and has been shown to have good robustness against outliers [14]:

$$
\Delta\left(i, j, k, d_{a}, d_{l}, d_{e}\right)=\left|g_{j}^{k}(i)-g_{j+d_{l}}^{f+d{ }^{\prime}}\left(i+d_{a}\right)\right|
$$

where the axial, lateral and elevational search ranges are limited by $d_{a, \min } \leq$ $d_{a} \leq d_{a, \max }, d_{l, \min } \leq d_{l} \leq d_{l, \max }$ and $d_{e, \min } \leq d_{e} \leq d_{e, \max }$.

$R\left(d_{a_{i}}, d_{l_{i}}, d_{e_{i}}, d_{a_{i-1}}, d_{l_{i-1}}, d_{e_{i-1}}\right)=\left(d_{a_{i}}-d_{a_{i-1}}\right)^{2}+\left(d_{l_{i}}-d_{l_{i-1}}\right)^{2}+\left(d_{e_{i}}-d_{e_{i-1}}\right)^{2}$

is the smoothness regularization. The cost function at each point $i, j$ and $k$ is

$$
\begin{aligned}
& C_{j}^{k}\left(d_{a}, d_{l}, d_{e}, i\right)=\Delta\left(i, j, k, d_{a}, d_{l}, d_{e}\right)+w_{1} R\left(d_{a}, d_{l}, d_{e}, d_{a}^{k-1}, d_{l}^{k-1}, d_{e}^{k-1}\right) \\
& +\min _{\delta_{a}, \delta_{l}, \delta_{e}}\left\{\frac{C_{j}^{k}\left(\delta_{a}, \delta_{l}, \delta_{e}, i-1\right)+C_{j-1}^{k}\left(\delta_{a}, \delta_{l}, \delta_{e}, i\right)}{2}+w_{2} R\left(d_{a}, d_{l}, d_{e}, \delta_{a}, \delta_{l}, \delta_{e}\right)\right\}
\end{aligned}
$$


where $w_{1}$ is a weight for governing smoothness in the elevational direction and $w_{2}$ is a weight for governing axial and lateral smoothnes 1 . Generally, the optimum values of $\delta_{a}, \delta_{l}, \delta_{e}$ should be sought in the entire $\left[\begin{array}{ll}d_{a, \min } & d_{a, \max }\end{array}\right] \times$ $\left[\begin{array}{ll}d_{l, \min } & d_{l, \max }\end{array}\right] \times\left[\begin{array}{ll}d_{e, \min } & d_{e, \max }\end{array}\right]$ space. However, since the strain value is low in elastography, it is expected and desired that at each sample of RF data, the change between the displacement of a sample and its previous sample is not more than 1 . Therefore, the search range is limited to the nine values of $\left\{d_{a}-1, d_{a}, d_{a}+1\right\} \times\left\{d_{l}-1, d_{l}, d_{l}+1\right\} \times\left\{d_{e}-1, d_{e}, d_{e}+1\right\}$, which results in a significant gain in speed. This limit on the search range does not affect the results even in a high strain of $10 \%$ : $\Delta d$ is zero for nine samples and one for the tenth sample on average. For memoization [12, $\delta_{a}, \delta_{l}$ and $\delta_{e}$ values that minimize the cost function are stored:

$$
\begin{aligned}
& M_{j}^{k}\left(i, d_{i}, d_{l}, d_{e}\right)= \\
& \arg \min _{\delta_{a}, \delta_{l}, \delta_{e}}\left\{\frac{C_{j}^{k}\left(\delta_{a}, \delta_{l}, \delta_{e}, i-1\right)+C_{j-1}^{k}\left(\delta_{a}, \delta_{l}, \delta_{e}, i\right)}{2}+w_{2} R\left(d_{a}, d_{l}, d_{e}, \delta_{a}, \delta_{l}, \delta_{e}\right)\right\}
\end{aligned}
$$

The cost function $C_{j}^{k}$ is calculated for $i=1 \cdots m, d_{a}=d_{a, \min } \cdots d_{a, \max }, d_{l}=$ $d_{l, \min } \cdots d_{l, \max }$ and $d_{e}=d_{e, \min } \cdots d_{e, \max }$. The minimum cost at $i=m$ gives the displacement of this point, which is traced back to $i=1$ using the $M$ function to calculate the three axial, lateral and elevational displacements $\left(D=\left(d_{a}, d_{l}, d_{e}\right)\right)$ :

$$
\begin{aligned}
& D_{j}^{k}(i)=\arg \min _{d_{a}, d_{l}, d_{e}}\left\{C_{j}^{k}\left(d_{a}, d_{l}, d_{e}, i\right)\right\}, i=m \\
& D_{j}^{k}(i)=M\left(i+1, D_{j}^{k}(i+1)\right), \quad i=1 \cdots m-1
\end{aligned}
$$

This gives all three displacements simultaneously, in contrast with other 3D elastography methods which give displacement in each direction in separate steps.

Further speed-up is achieved by downsampling the signal $g(i)$ in the axial direction by a factor of $\beta$ to $g^{*}(i)$, and comparing it with the unaltered signal $g^{\prime}(i)$. This is done by simply skipping $\beta-1$ samples from $g(i)$ and performing DP on the $\beta^{\text {th }}$ sample as illustrated in Figure 1 left. This generates integer displacement estimations at $m / \beta$ samples. The displacement of the skipped samples is then simply approximated by the linear interpolation of two neighboring points whose displacements are calculated, as an initial guess for the next step.

The displacement estimates are then refined to subpixel displacement estimation in the axial direction. The original signal $g(i)$ (not downsampled) is compared with $g^{\prime}(i+d)$ upsampled by a factor of $\gamma$ (Figure 1 right) in the axial

$\overline{1}$ The inclusion of the cost of the previous line $\left(C_{j-1}^{k}(\cdots)\right)$ guarantees lateral smoothness. Instead, we could force the displacements of each pixel to be similar to the displacements of the neighboring pixel in the previous A-line, similar to what we did in the $w_{1} R(\cdots)$ term to enforce elevational smoothness. The former is preferred since a wrong displacement estimation does not affect the neighboring A-line's displacement estimation. However, it requires the $C_{j-1}^{k}(\cdots)$ to be kept until the calculation of $C_{j}^{k}(\cdots)$ is completed. Therefore, at each time only two cost finctions are stored in the memory, making the memory requirement independent of the number of A-lines. 


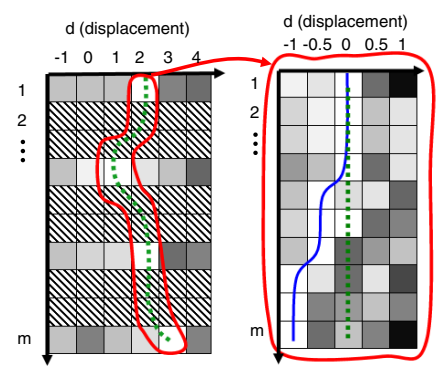

Fig. 1. In the left, the cost function $C$ is shown when DP is performed on $g^{*}(i)(g(i)$ downsampled by a factor of $\beta$ ) and $g^{\prime}(i)$ (not downsampled). Hashed squares indicate no cost calculation is performed due to downsampling of $g(i)$, and white and black representing low and high cost values respectively. Displacement is calculated at $\mathrm{m} / \beta$ samples in this stage $(\beta=3$ in this figure). In right, a new cost function around the optimum path of the first stage's cost function (the dashed line) is created, giving a $1 / \gamma=1 / 2$ pixel displacement accuracy at $m$ samples.

direction using parabolic interpolation. Repeating the refinement procedure $n$ times results in a refinement factor of $1 / \gamma^{n}$. The code runs in approximately $30 \mathrm{sec}$ for a typical volume on a $3.8 \mathrm{GHz} \mathrm{P} 4 \mathrm{CPU}$.

In cross correlation methods, subsample displacement is usually achieved by interpolation of the correlation function, which is subject to bias and jitter [15]. Here we interpolate the original RF data instead, which is shown to have similar performance [15]. Although cosine-fit outperforms parabolic-fit interpolation in terms of bias and jitter [15], the latter is used here for computational simplicity.

\section{Results}

We first present in-vivo elastographic monitoring of RF ablation therapy of $\mathrm{HCC}$ in human during surgery using 2D DP elastography. RF ablation was administered using the RITA Model 1500X RF generator (Rita Medical Systems, Fremont, CA). Ultrasound RF data is acquired from an Antares Siemens system (Issaquah, WA) with a $7.27 \mathrm{MHz}$ linear array at a sampling rate of $40 \mathrm{MHz}$. Figure 2 shows the B-mode scan, the strain image obtained using the DP method and CT scans performed after RF ablation (first and second row corresponding to first and second patient respectively). Tissue is simply compressed freehand with the ultrasound probe without any attachment. The shadow in Figure 2(a) at $20 \mathrm{~mm}$ depth is produced by the thermal lesion. Note that it is not possible to ascertain the size and position of the thermal lesions from B-mode images. In addition, the thermal lesion has different appearances in the two B-scans. However, the thermal lesions show very well as hard lesions in both the strain images. The size of the thermal lesion in the strain images and in CT scans are also in accordance. The strain images provide with higher contrast of the thermal lesion and lower noise in the image, compared to the strain images of RF ablation 


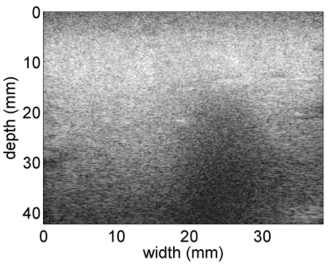

(a)

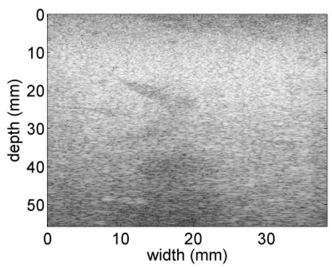

(d)

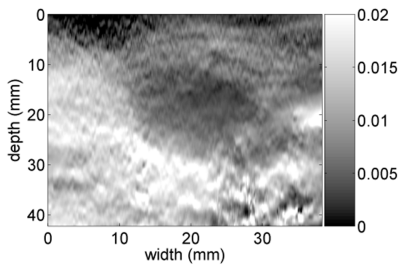

(b)

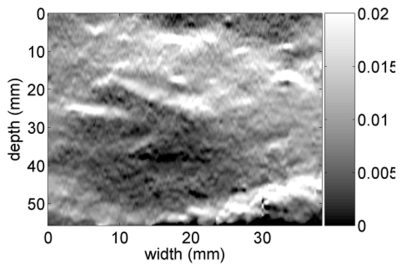

(e)

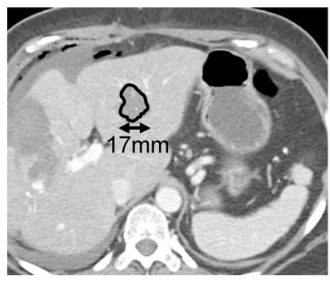

(c)

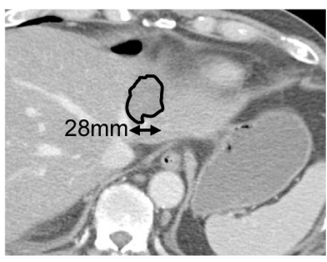

(f)

Fig. 2. In-vivo images of the thermal lesion produced by RF ablation therapy of HCC, first and second row corresponding to the first and second patients. (a) \& (d) Bscan after RF ablation. The shadow in (a) indicates the presence of thermal lesion. It is almost impossible to ascertain the size and position of the thermal lesion from the B-scans. (b) \& (e) Strain images after RFA ablation, generated using 2D DP elastography and freehand palpation of the liver tissue. The thermal lesion is visible in dark surrounded by normal tissue in white. (c) \& (f) Post-ablation CT scans, with the delineated thermal lesions (The non-unity aspect ratio in the axes of the B-mode and strain images should be considered when comparing them with the CT scans).

reported in 38] which are obtained with cross-correlation. To the best of our knowledge, this is also the first demonstration of the success of elastography in imaging the thermal lesion in an in-vivo human experiment.

A Radionics device (Valleylab, Boulder, CO) is used for ex-vivo RF ablation. For 3D elastography, we use a 3D probe that consists of a curvilinear array that is mechanically rotated to scan a volume. Ultrasound RF data is acquired from an Ultrasonix system (Vancouver, BC) at $4.5 \mathrm{MHz}$ frequency, $20 \mathrm{MHz}$ sampling rate and 30\% bandwidth. Figure 3 shows the experimental setup and results. Comparing strain images obtained during ablation and after ablation, the growth of the thermal lesion can be observed. There is also a good agreement between the size of the lesion in axial and lateral directions in the post-ablation strain images and gross pathology photograph. The ablation goes beyond the field of view of the $3 \mathrm{D}$ ultrasound probe in the elevational direction. The volumetric elastography contrast to noise ratio (CNR) [2] $\left(\mathrm{CNR}=\sqrt{\frac{2\left(\bar{s}_{b}-\bar{s}_{t}\right)^{2}}{\sigma_{b}^{2}+\sigma_{t}^{2}}}\right.$ where $\bar{s}_{t}$ and $\bar{s}_{b}$ are the spatial strain average of the target and background and $\sigma_{t}^{2}$ and $\sigma_{b}^{2}$ are the spatial strain variance of the target and background) between two $3 \mathrm{~mm} \mathrm{x}$ $3 \mathrm{~mm} \times 3 \mathrm{~mm}$ cubes, one in the thermal lesion and the other half way between the liver surface and the lesion, is 3.4. Note that due to the lateral and elevational 


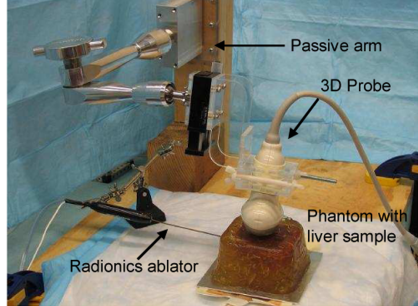

(a)

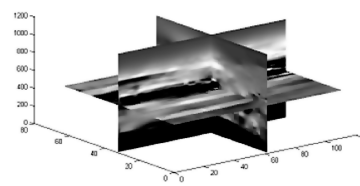

(c)

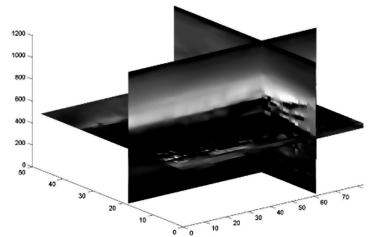

(g)

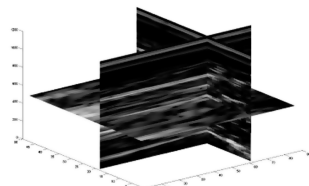

(k)

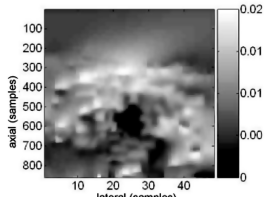

(d)

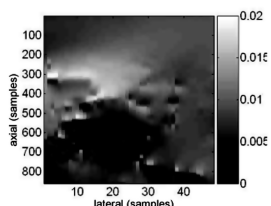

(h)

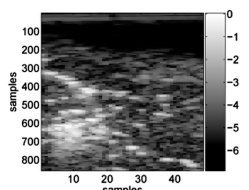

(1)

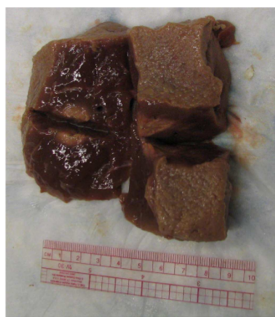

(b)

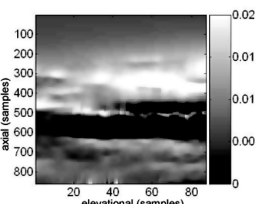

(e)

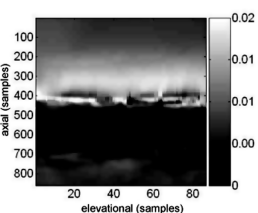

(i)

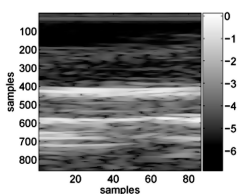

(m)

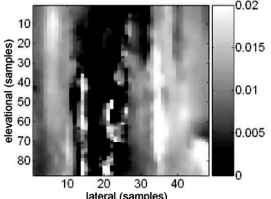

(f)

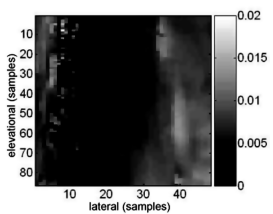

(j)

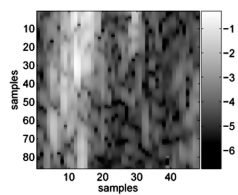

(n)

Fig. 3. Ex-vivo liver RF ablation experiment. The ablation power is set to $8 \mathrm{~W}$ for $10 \mathrm{~min}$ and the cooler is turned off throughout the experiment. (a) The experimental setup. The passive arm is holding the $3 \mathrm{D}$ probe and the liver is contained in the gelatin phantom. (b) The liver sample after ablation (cut into four pieces) with the thermal lesion. (c)-(f) 3D strain images $6 \mathrm{~min}$ after the start of the ablation (target temperature reached $90^{\circ} \mathrm{C}$ in $3 \mathrm{~min}$ and was approximately constant in the next $7 \mathrm{~min}$ of ablation). (g)-(j) 3D strain images after the ablation. (k)-(n) 3D B-mode images after the ablation. Each 650 axial samples correspond to $25 \mathrm{~mm}$ in the strain and B-mode images. Each 40 lateral samples correspond to $16.6 \mathrm{~mm}$ and $30.4 \mathrm{~mm}$ on the top and bottom of each image (different values on the top and bottom since the probe is curved). Each 80 elevational samples correspond to $3.4 \mathrm{~mm}$ and $6.3 \mathrm{~mm}$ on the top and bottom of each image.

regularization, DP elastography is working reasonably in the presence of the ablation needle. 


\section{Discussion and Conclusion}

Previous work has shown promise in monitoring ablation in 2D ex-vivo and animal experiments. In this paper, we present high quality in-vivo 2D strain images of thermal lesions and compared them to post-ablation CT data. Comparison is more qualitative, however, since strain images are $2 \mathrm{D}$ and $\mathrm{CT}$ data is $3 \mathrm{D}$ and ultrasound is not tracked. We also present formulation and experimental results of a 3D strain imaging system based on DP. In DP, we regularize the problem of $3 \mathrm{D}$ displacement estimation: regularization in $2 \mathrm{D}$ is shown to increase robustness [6]. As a result, no post processing step such as median filtering is performed.

We demonstrate the feasibility of 3D elastography monitoring of RF ablation for the first time using a 3D probe; however, we are planning for a comprehensive comparison of the 3D DP with other 3D strain imaging techniques [10/11]. The lateral and elevational search is performed only to increase the quality of the axial strain: the lateral and elevational displacements are integer values and are not suitable for calculating strain. Good volumetric CNR between the thermal lesion and background suggests that the regularization is not adversely affecting CNR. However, a study similar to [6] on the effect of the 3D regularization on the CNR and resolution should be done. Having an elastography system for 3D ablation monitoring with promising ex-vivo results, in-vivo patient studies under our active Institutional Review Board (IRB) approval are to commence.

\section{References}

1. Rossi, S., Di-Stasi, M., Buscarini, E., et al.: Percutaneous RF interstitial thermal ablation in the treatment of hepatic cancer. AJR 167, 759-768 (1996)

2. Righetti, R., et al.: Elastographic characterization of hifu-induced lesions in canine livers. Ultras. Med. Biol. 25, 1099-1113 (1999)

3. Varghese, T., et al.: Elastographic imaging of thermal lesions in the liver in vivo following radiofrequency ablation: prelim results. US Med. Biol. 28, 1467-1473 (2002)

4. Varghese, T., et al.: Tradeoffs in elastographic imaging. US. Imag. 23, 216-248 (2001)

5. Xie, H., et al.: The role of local center frequency estimation in doppler-based strain imaging. In: IEEE Ultrasonics Symp., New York, NY, pp. 1965-1968 (2007)

6. Rivaz, H., Boctor, E., et al.: Ultrasound elastography: a dynamic programmimg approach. IEEE Trans. Medical Imaging (in press)

7. Krueger, M., et al.: Ultrasonic strain imaging of the female breast using phase root seeking and three-dimensional "optical flow". IEEE US Symp. 60, 1757-1760 (1998)

8. Liu, W., Zagzebski, J., Varghese, et al.: Segmentation of elastographic images using a coarse-to-fine active contour model. Ultrasound Med. Biol. 32, 397-408 (2006)

9. Lindop, J., et al.: 3D elastography using freehand ultrasound. Ultrasound Med. Biol. 32, 529-545 (2006)

10. Treece, G., et al.: Freehand elastog using a 3-D probe. US Med. Biol. (in press)

11. Fisher, T., et al.: Volumetric strain imaging. In: IEEE US Symp., pp. 355-358 (2007) 
12. Bellman, R.: Applied dynamic programming. Princeton U. Press (1962)

13. Brown, M., et al.: Adv in computational stereo. IEEE PAMI 25, 993-1008 (2003)

14. Huber, P.: Robust statistics. John Wiley \& Sons, Chichester (1981)

15. Viola, F., Walker, W.: A spline-based algorithm for continuous time-delay estimation using sampled data. IEEE Trans. Ultras. Ferr. Freq. Cont. 52, 80-93 (2005) 